Bayo Guardiola, R. y Moliner García, O. (2021). Alumnado con enfermedades poco frecuentes (EPF) en las aùlas ordinarias: ¿cómo se garantiza su presencia, participación y aprendizaje?. Revista de Investigación Educativa, 39(2), 571-586.

DOI: http://dx.doi.org/10.6018/rie.449011

\title{
Alumnado con enfermedades poco frecuentes (EPF) en las aulas ordinarias: ¡cómo se garantiza su presencia, participación y aprendizaje?
}

\section{Rare disease students in regular classrooms: how is their presence, participation and learning guaranteed?}

\author{
Rosa Bayo Guardiola y Odet Moliner García' \\ Universitat Jaume I
}

\begin{abstract}
Resumen
El presente estudio forma parte de un estudio más amplio sobre enfermedades poco frecuentes (EPF) e inclusión educativa. La respuesta educativa al alumnado con enfermedades poco frecuentes, también denominadas raras, minoritarias o de baja prevalencia, implica un abordaje integral desde el ámbito educativo, sanitario y psicosocial que supone un reto para los procesos de inclusión que se desarrollan en muchas escuelas. El objetivo de esta investigación es analizar cuáles son los procesos educativos que favorecen la inclusión del alumnado con enfermedades poco frecuentes en las aulas ordinarias. La metodología de estudio de casos múltiples, permite analizar en profundidad los procesos inclusivos desarrollados en tres centros educativos ordinarios de la Comunidad Valenciana que escolarizan alumnado con EPF. Se trata de una investigación cualitativa que recoge la voz de equipos directivos, personal docente y no docente, familiares y alumnado con EPF mediante entrevistas y grupos focales. Los resultados muestran qué procesos desarrollados en las escuelas garantizan la presencia, participación y aprendizaje del alumnado con EPF. Las conclusiones ponen de relieve la importancia de la planificación y la coordinación de los agentes implicados, la organización de los apoyos, sobre todo los específicos, y abre la discusión sobre los dilemas que plantea la evaluación de los aprendizajes.

Palabras clave: enfermedades raras; enfermedades poco frecuentes; educación inclusiva; aulas ordinarias.
\end{abstract}

Correspondencia: Odet Moliner García, molgar@uji.es, Universitat Jaume I 


\begin{abstract}
This study is part of a larger study on rare diseases (RD) and educational inclusion. The educational response to students with rare diseases, also known as rare, minority or low prevalence diseases, implies a comprehensive approach from the educational, health and psychosocial fields that challenges the inclusion processes that are developed in many schools. The aim of this research is to analyse which educational processes favour the inclusion of students with rare diseases in ordinary classrooms. The methodology of multiple case studies allows for an in-depth analysis of the inclusive processes developed in three ordinary schools in the Valencian Community that educate students with $R D$. This is a qualitative study that brings together the voices of management teams, teaching and non-teaching staff, families and students with $R D$ through interviews and focus groups. The results show which processes developed in the schools guarantee the presence, participation and learning of the students with RD. The conclusions highlight the importance of the planning and coordination of the agents involved, the organisation of support, especially specific support, and open up the discussion on the dilemmas posed by the evaluation of learning.

Keywords: rare diseases; infrequent diseases; inclusive education; regular classrooms.
\end{abstract}

\title{
Introducción
}

Afortunadamente la inclusión está siendo el motor de las reformas educativas de muchos países. Ello implica que para universalizar esta declaración de principios hay que avanzar en políticas, prácticas e investigaciones educativas en términos de inclusión (Bartolomé, 2017) y esto, en algunos contextos, no es fácil. En la mayoría de los países europeos, a excepción de Italia, la educación especial continúa siendo una alternativa a la general y ordinaria (Linertová et al., 2019) lo cual constituye dos sistemas paralelos que nos alejan de la escuela inclusiva como espacio social en el que todas las personas caben.

La escolarización del alumnado con EPF en contextos ordinarios supone todo un reto por la complejidad de hacer converger en un contexto ordinario respuestas que atiendan a sus necesidades sanitarias, sociales y educativas. También son escasos los estudios sobre prácticas de inclusión y cuando existen se trata de prácticas puntuales y no están sistematizadas.

Pero, ¿qué entendemos por alumnado con EPF? Este es el término que adoptamos en este estudio, asumiendo la propuesta del Ministerio de Sanidad, cuando en 2014 propuso cambiar el nombre de enfermedades raras por el de enfermedades minoritarias o poco frecuentes (EPF) con el propósito de evitar la connotación peyorativa que conlleva el adjetivo 'raro' (Monzón y Gaintza, 2014).

Justamente la baja prevalencia de algunas enfermedades es lo que define su consideración de EPF. En Europa, según la Red Epidemiológica de Investigación de Enfermedades Raras (REpIER) (Aróstegui y Monzón, 2017), una enfermedad se considera poco frecuente si su prevalencia es igual o menor a 5 casos cada 10.000 personas, y si su etiología y tratamiento es desconocido o indefinido. Estos datos equivalen a un 6-8\% de la población europea, aproximadamente 29 millones de personas en Europa, 
y 3 millones a España, es decir, entre un 6 y un 7\% de los habitantes (Ministerio de Sanidad y Política Social, 2009). La diversidad en el origen, los síntomas y la complejidad de este conjunto de enfermedades, juntamente con la falta de conocimiento y el uso de criterios no universales, impide determinar con precisión el número de personas afectadas (Posada, Martín-Arribas, Ramírez, Villaverde y Abaitua, 2008). Por ejemplo, la prevalencia mencionada de la Unión Europea (UE) es diferente a la de los Estados Unidos, donde se utiliza una cifra global de casos, menos de 200.000 en todo el país (Solves, Arcos, Páramo, Sánchez y Rius, 2018). La heterogeneidad de las patologías de las personas que padecen EPF es la causa de conformar un colectivo segregado y vulnerable con unas necesidades específicas que requieren ser atendidas (Linertová et al., 2019). Esa atención pasa por realizar un seguimiento multidisciplinar para responder a sus condiciones motoras, sensoriales y cognitivas (Posada et al., 2008; Solves et al., 2018). Aun así, la respuesta social que reciben no es suficiente para poder satisfacer sus derechos como ciudadanía (Orcasitas y Aróstegui, 2017).

La Comisión del Grupo Experto en Enfermedades Raras de la UE (CEG-RD, 2016) señaló la importancia de abordar, eliminar y prevenir las barreras del entorno que dificultan la participación de las personas con diversidad funcional a causa de su EPF en la sociedad, en igualdad con otros ciudadanos, sobre todo en el ámbito educativo, empleo, actividades de la vida diaria, accesibilidad y movilidad.

El principio de la educación inclusiva de todo el alumnado es introducido por primera vez en la legislación española en la LOE (2006). Sin embargo, han tenido que pasar casi 15 años para que algunas comunidades autónomas como Cataluña o la Comunidad Valenciana, promulguen sus respectivos decretos autonómicos de inclusión. En España, un estudio sobre las necesidades sociosanitarias de las personas con EPF (Solves et al., 2018) presentó que el 41,30\% de este colectivo se había sentido alguna vez discriminado en el ámbito educativo, una cifra mayor al 29,84\% del 2009. Estos datos cuestionan la garantía de la igualdad de oportunidades a la educación que establece la legislación. Aunque hoy en día el derecho a la educación inclusiva no es cuestionado por nadie, la normativa ampara tímidamente la situación particular de las personas con EPF. Por ejemplo, en el Decreto 104, 2018 de la Comunidad Valenciana se establece, entre otras, la prestación de apoyos al alumnado que no pueda asistir al centro educativo por situaciones de enfermedad.

En un trabajo coordinado por Gaintza y Ozerinjauregi (2017) sobre experiencias de escolarización del alumnado con EPF, se pone de manifiesto la necesidad de desarrollar estrategias metodológicas y organizativas del centro para adaptarse a los ritmos de aprendizaje y las posibles ausencias, como consecuencia de la enfermedad ((Moliner y Sales, 2017). La coordinación interdisciplinar y la buena comunicación entre las redes educativas, sanitarias y sociales que atienden al alumnado con EPF es otra de los factores indispensables (Aróstegui y Monzón, 2017). Además, es importante la comunicación bidireccional entre las familias y el centro, con una actitud de colaboración desde el respeto, el cuidado y la escucha (Ledesma y Pellejero, 2017), así como la eliminación de barreras para ofrecer al alumnado una mayor autonomía y la posibilidad de compartir espacios y experiencias entre sus iguales. También la formación docente y del alumnado para la aceptación de la diversidad y la visibi- 
lidad de las EPF es un factor determinante para su inclusión (Verger, De la Iglesia, Paz-Lourido y Negre, 2017).

Todos estos elementos son clave para aseguran la presencia, la participación y el éxito del alumnado con EPF en los centros educativos ordinarios, independientemente de sus características y desde un enfoque inclusivo. Considerando las aportaciones de Ainscow et al. (2006) la primera condición para la inclusión es la presencia de todo el alumnado, en todas las experiencias de aprendizaje que se desarrollan en el grupo de referencia (Muntaner, Rosselló y De la Iglesia, 2016). La segunda condición es la participación de todo el alumnado, en todas las situaciones de aprendizaje respetando sus capacidades y potencialidades. La tercera y última condición es el progreso, éxito o logro, y exige que todo el alumnado aprenda, dentro de sus posibilidades, en todas las actividades y situaciones.

Aunque la revisión de la literatura pone de manifiesto las escasas investigaciones que existen sobre la escolarización inclusiva del alumnado con EPF en el ámbito internacional (Linertova et al., 2019), en nuestro contexto, existen algunos estudios que ponen el foco en las necesidades y las propuestas para la inclusión en las escuelas ordinarias (García Parra, 2019; Monzón, Aróstegui y Ozerinjauregi, 2017; Monzón y Gaintza, 2014; Muntaner, Forteza y Salom, 2014).

El siguiente estudio forma parte del proyecto Inkluni, una investigación nacional de carácter cualitativo liderado por la Universidad del País Vasco en colaboración con universidades de diez de comunidades autónomas y FEDER (Federación Española de Enfermedades Raras) cuyo objeto es analizar y sistematizar buenas prácticas educativas que responden a las necesidades del alumnado con EPF.

\section{Método}

\section{Pregunta y objetivo de la investigación}

Este estudio responde a la pregunta: ¿qué procesos facilitan la inclusión del alumnado con enfermedades poco frecuentes en los centros educativos ordinarios? El objetivo es analizar cuáles son los procesos inclusivos que promueven la presencia, participación y aprendizaje del alumnado con EPF escolarizado en tres centros educativos de la Comunidad Valenciana. Se trata de conocer e interpretar qué elementos clave garantizan su inclusión en estos centros, desde las perspectivas de las personas implicadas en cada caso.

\section{Diseño}

El diseño metodológico, de carácter cualitativo, es un estudio de casos múltiples, de carácter instrumental (Stake, 2010) porque su análisis pretende analizar y comprender tres casos particulares dentro de la singularidad y complejidad de cada contexto y la interrelación entre los protagonistas y su entorno (Bisquerra, 2009; Stake, 2010). Conocer la perspectiva de los estudiantes con EPF, sus familias y de los profesionales de 
cada centro, permite interpretar qué elementos garantizan su presencia, participación y aprendizaje en las aulas.

\section{Selección de casos y contextualización}

Para la selección de los tres centros educativos del estudio de casos, se creó un Equipo Asesor Ético (EAE) compuesto de 6 personas: 1 familiar de un joven con EPF, 2 representantes del Centro de Formación Innovación y Recursos Educativos (CEFIRE),1 representante de FEDER en la Comunidad Valenciana y 2 miembros del grupo de investigación. Tras una primera reunión de presentación del proyecto, los miembros del EAE aportaron diversos casos, que fueron valorados en función de los siguientes criterios de selección: a) que escolarizaran alumnado con EPF, b) que sus familiares se mostraran satisfechos con las prácticas inclusivas, c) que el centro contara con un proyecto de inclusión o de atención a la diversidad sostenido en el tiempo. Así, la selección fue intencional y el equipo tuvo en cuenta también la accesibilidad al centro y a las familias de los niños con EPF. En cuanto al contexto de los centros, el primer caso, CAS1, es una escuela pública de educación infantil y primaria de la provincia de Castellón situada en un contexto socioeconómico medio. Cuenta con 255 alumnos, un $25 \%$ de los cuales tienen un diagnóstico de necesidades específicas de apoyo educativo. Dentro de la filosofía del centro destaca su proyecto sobre educación emocional e inclusiva para la convivencia y el éxito escolar de todo el alumnado. Este enfoque favorece también la inclusión de una niña de 8 años de tercero de primaria, diagnosticada de síndrome de Williams. Se trata de una EPF que afecta a 1 de cada 20.000 personas $^{1}$ que comporta un trastorno del neurodesarrollo, que afecta a la comunicación, la vida social y la autonomía.

El segundo centro, CAS2, también es una escuela pública de educación infantil y primaria de la provincia de Castellón situada en un contexto socioeconómico bajomedio. Es un centro de atención preferente de alumnado con dificultades motoras al que asisten 400 alumnos, con un amplio abanico de alumnado con diversidad funcional. El proyecto de centro contiene un Plan de Inclusión, cuyo propósito es atender a todo el alumnado en las aulas ordinarias. La niña de 7 años de primero de primaria con Síndrome de Rett, EPF que afecta a 1 de cada 15.000 personas y conlleva un retraso global del desarrollo cognitivo y motor, está muy presente en su grupo clase.

El CAS3 es un instituto público de educación secundaria y bachillerato de Valencia, situado en un contexto socioeconómico medio-alto. Al mismo asisten 350 alumnos, con un número considerable de alumnado con altas capacidades y recién llegado. El centro cuenta con un proyecto de convivencia que, desde la figura de la coordinadora de igualdad, fomenta una actitud positiva hacia la diversidad. Al centro asiste un joven de 16 años con Fiebre Mediterránea Familiar, patología que afecta de 1 a 5 personas de cada 10.000 y se caracteriza por episodios de cansancio y dolor.

1 Datos extraídos de Orphanet. (2020). The portal for rare diseases and orphan drugs. https://www.orpha.net/ consor/cgi-bin/Disease_Search.php?lng=EN 


\section{Recogida de información: instrumentos y participantes}

El acceso al centro, al profesorado y a las familias fue confirmado y facilitado por la dirección en los tres casos. La recogida de información se realizó mediante entrevistas individuales (EI) y grupos focales (GF) (Bisquerra, 2009) con el objetivo de garantizar la triangulación metodológica, contribuyendo así a la fiabilidad y validez de los datos obtenidos. Teniendo en cuenta aspectos éticos de la investigación, se firmaron los correspondientes consentimientos informados para confirmar el interés y compromiso de los informantes en el proceso de investigación (Parrilla, 2010). Se utilizaron como instrumentos de recogida de información tres guiones de entrevista semiestructurada, dirigidos a recoger la voz de cada sector: uno para las familias y alumnado, otro para la dirección del centro y el tercero para los profesionales implicados. Los guiones se elaboraron a partir de las preguntas de reflexión de la guía Themis (Azorín y Ainscow, 2018), una herramienta inspirada en el Index for Inclusion (Booth y Ainscow, 2011), que orienta a los centros educativos en la valoración y la mejora de la inclusión. En este estudio se indaga en la dimensión $\mathrm{C}$, procesos inclusivos.

En el estudio participaron 24 personas: 1 alumno (A), 3 madres (M), 3 directores (D), 2 jefes de estudios (JE), 3 tutores (T), 2 educadoras (ED), 1 fisioterapeuta (F), 3 especialistas de audición y lenguaje (ALE), 3 de pedagogía terapéutica (PT), 1 orientador $(\mathrm{O})$ y 2 maestros especialistas $(\mathrm{ME})$. Se realizaron 8 entrevistas individuales (EI 1-8) y 6 grupos focales (GF 1-6), como puede verse en la tabla 1.

Tabla 1

Participantes en las entrevistas individuales (EI) y grupos focales (GF).

\begin{tabular}{ccccccc}
\hline Caso & \multicolumn{5}{c|}{ Técnica y Participantes } \\
& \multicolumn{5}{c}{ GF2: PT y ALE } \\
CAS1 & EI1: $M$ & EI2: D & $\begin{array}{c}\text { GF1: T, ME1 } \\
\text { y ME2 }\end{array}$ & GF \\
CAS2 & EI3: $M$ & EI4: D & EI5: T & $\begin{array}{c}\text { GF3: D, JE, F, ED1, ED2, } \\
\text { PT1, PT2, ALE1 y ALE2 }\end{array}$ \\
CAS3 & EI6: $M$ & $\begin{array}{c}\text { GF4: } D \\
\text { y JE }\end{array}$ & EI7: T & EI8: PT & GF5: O & GF6: A \\
& & & & y M & y M \\
\hline
\end{tabular}

\section{Procedimiento del análisis de datos}

Una vez recogida toda la información, se procedió a la transcripción literal de las entrevistas de cada estudio de caso. En esta fase, bajo el criterio ético de la investigación (Parilla, 2010), se devolvió la información a los informantes que aprobaron que se hiciera uso de sus datos. El análisis de contenido se realizó con el apoyo del Nvivo, versión 12, un programa informático con un sistema categorial que agilizó la sistematización de datos cualitativos. El análisis fue deductivo, y se codificaron un total de 343 unidades de significado a partir las categorías y subcategorías teóricas de la dimensión C de la herramienta Themis (Azorín y Ainscow, 2018), tal y como se recoge en la Tabla 2. 
Tabla 2

Categorías y subcategorías

\begin{tabular}{l|l|}
\hline Categorías & \multicolumn{1}{c|}{ Subcategorías } \\
\hline Presencia (C1) & C.1.1. Planificación de la enseñanza \\
& C.1.2. Variedad metodológica \\
Participación (C2) & C.2.1. Tipo de agrupamiento \\
& C.2.2. Organización espacios y tiempos \\
& C.2.3. Apoyo \\
Logro (C3) & C.3.1. Evaluación \\
& C.3.2. Tránsito \\
\hline
\end{tabular}

\section{Resultados}

La presentación de los resultados se organiza siguiendo la estructura de las tres categorías de primer nivel (presencia, participación y logro). En cada apartado se muestran los resultados del segundo nivel de análisis, en función de las subcategorías teóricas y emergentes. Un mapa relacional (elaborado con la herramienta CMaps Tools) permite visualizar y poner en relación los elementos clave señalados en los tres casos. Estos se presentan, en coherencia con el método del estudio, ilustrados con los verbatim literales de los informantes y haciendo referencia a las particularidades de cada caso.

\section{Presencia}

Como puede apreciarse en la figura 1, para garantizar la presencia, los informantes consideran importante la planificación de la enseñanza (C.1.1.) que tiene como finalidades la coordinación, la evaluación o la organización de horarios, decisiones que se plasman en los documentos de centro. En el caso 1 y 2 se insiste en la coordinación y priorización de los objetivos de aprendizaje que hay que trabajar con el alumnado.

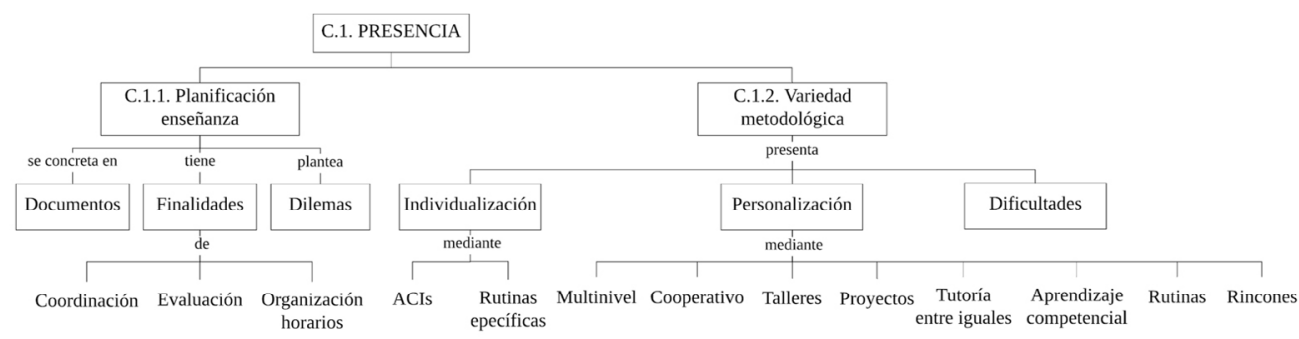

Figura 1. Mapa relacional: presencia 
En el CAS1 los objetivos a trabajar con la niña con EPF se eligen con la finalidad de "reforzarle entre todas ese aspecto curricular que ella tanta dificultad tiene y tanta falta le hace a la hora de poder estar presente en las actividades que realiza" (GF2, ALE) en el aula.

En el CAS2 la planificación de los objetivos surge de una evaluación previa: "lo que hacemos al inicio de curso, es una coordinación: evaluamos items y acordamos items de trabajo, de forma que todos trabajamos los mismos items con ella" (GF3, ALE1). El éxito de la planificación, para el personal docente y la familia consiste en ir todos a una, "las reuniones que se hacen para programar los objetivos y la metodología, todo se hace en conjunto. No lo hago yo sola, ni los especialistas solos. Y al conjunto, me refiero también a la familia y externos, gabinetes externos" (EI5, T).

En ambos casos las necesidades del alumnado son un aspecto clave cuando se planifica la organización de espacios y tiempos (C.2.2.) y los apoyos (C.2.3.), mencionados en el siguiente apartado. "En función de las necesidades que tiene la niña, decidimos qué sesiones serán más provechosas que pudiéramos entrar nosotras en el aula" (CAS1, GF2, ALE).

La planificación también genera dilemas, algunos relacionados con la evaluación, como en el CAS3 cuando el tutor, el orientador, el director y la PT se plantean "intentar unificar criterios, aspectos metodológicos, de tratar no solo al alumnado con necesidades, sino a todo el grupo" (GF4, D). Un cambio necesario, admiten, para evitar el uso de las ACIs (Adaptaciones Curriculares Individuales Significativas) que ven como "parches" de un currículo segregado que condiciona el fracaso escolar:"ellos tienen unos criterios que siguen con todo su grupo, si mi alumno no llega a esos mínimos, ellos no lo quieren aprobar" (EI8, PT).

Por otra parte, la variedad metodológica (C.1.2.) es abordada desde dos perspectivas: la individualización y la personalización del aprendizaje. Por un lado, la individualización atiende las necesidades del alumnado con EPF, y a veces, implica priorizar o añadir otros contenidos distintos a los del grupo clase que se trabajan mediante ACIs o rutinas específicas. En el CAS1, "se intenta que la ACIs trabaje al nivel de la clase para que tampoco se sienta, por no tener el mismo material, desplazada" (EI2, D). Puesto que las niñas del caso 1 y 2 requieren de rutinas específicas para potenciar su autonomía, insisten en la cohesión y repetición de los hábitos en distintos espacios y tiempos:"Es una niña que necesita un trabajo muy rutinario para lograr aprendizajes" (CAS2, GF3, ALE1). Esto contrasta con el caso 3, ya que "no necesita adaptación curricular, por eso digo que nuestra enfermedad es muy fácil para hacer la adaptación" (GF6, M).

Por otro lado, la personalización del aprendizaje implica la diversificación metodológica para que todo el alumnado pueda estar presente. En el CAS1, afirman que en el aprendizaje cooperativo el beneficio es recíproco "esta niña también puede aportar mucho dentro de ese grupo, porque realmente los otros también están aprendiendo de ella, no ella solo aprende de los otros" (EI2, D). La interacción a través de los talleres o rincones es positiva pues "están haciendo a lo mejor cosas jugando, y ella claro, lo comprende más" (EI1, $\mathrm{M})$. También destacan la tutoría entre iguales, pues la alumna con EPF es muy buena en inglés: "le leo un fragmento en voz baja y ella repite, entonces a mí me sirve de modelo para los demás a nivel de 'listening"' (GF1, ME1). O en el CAS2 mediante la lectura entre iguales:

"Ella (la niña con EPF) siempre leía el título, un compañero leía el texto y otro compañero leía las preguntas del texto, y lo que hacíamos era que iban contestándolo y cada uno lo hacía a su nivel. Los compañeros iban contestando las preguntas y ella ponía el título, hacía un dibujo y ponía los nombres de los personajes." (GF2, ALE1). 
También en el CAS2, se aplican propuestas multinivel: "a ella (la niña con EPF) le intercalo muchas veces música, vídeo, imágenes, dibujos grandes...Que me sirven, en general, para todos y para ella también, porque constantemente la estoy teniendo en atención" (EI5, T).

En el CAS3 se comenta el aprendizaje competencial mediante exposiciones orales y proyectos con la intención de "aplicar una metodología constructivista, en la cual el alumnado adquiera las competencias haciendo cosas"(GF4, D).

Sin embargo, una dificultad encontrada es la resistencia al cambio metodológico en secundaria debido a que "cada profesor tiene su parcela autónoma" (GF5, O, M).

\section{C2. Participación}

Los procesos inclusivos que favorecen la participación del alumnado en el aula y en el centro se refieren al tipo de agrupamiento, a la organización de espacios y tiempos y a los apoyos previstos, tal y aparece en la figura 2

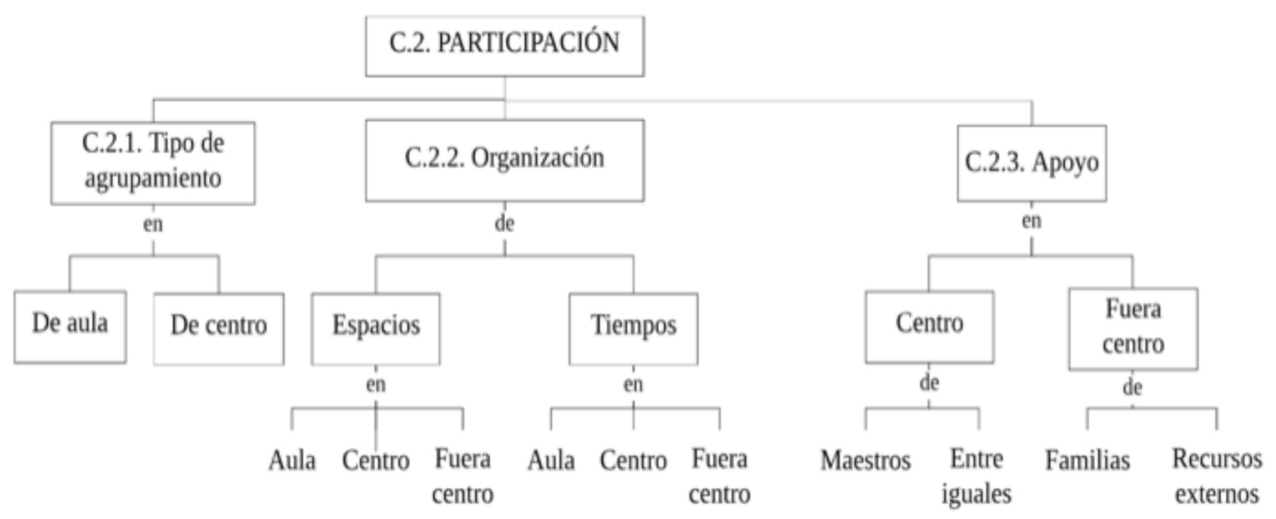

Figura 2. Mapa relacional: la participación

Los informantes afirman buscar un tipo de agrupamiento (C.2.1.) en el aula que busca la heterogeneidad. En el CAS1, consideran que ello permite poner en valor la diversidad como enriquecedora pues "ya no es que ella sea diferente es que todos son diferentes ¿no? Y todos tenemos habilidades, todos tenemos algunas cosas en las que necesitamos más ayuda" (GF2, PT). En el CAS2 "se ha ido cambiando constantemente (el agrupamiento) para que ella se acostumbre a todos los compañeros" (EI5, T). Y en el CAS3, el tutor tiene en cuenta las necesidades de aprendizaje de sus alumnos a la hora de distribuir el aula, para fomentar el apoyo entre iguales.

En la organización de espacios y tiempos (C.2.2), en el aula del CAS1, se valora la proximidad del docente al alumnado con necesidades para poder atenderle dentro de su grupo y ponerse al mismo nivel, sin estar encima de ellos. En este caso, "ella (la tutora) no tiene mesa de maestro, se sienta con el grupo y siempre la tiene muy cerca de ella (a la niña), coincidiendo muchas veces que también está la educadora" (EI2, D). En el CAS2, se plantea un cambio en la organización del espacio del aula para promover la participación de 
la niña: "me podría funcionar más si estructuro por rincones, y entonces querría hacer lo del trabajo autónomo, por equipos, individual con la mínima ayuda [ ] estará más dentro aún. La cuestión es romper con toda la estructura y cambiar" (EI5, T). Las maestras de PT del caso 2 y del 3 coinciden en que la organización de los espacios está limitada por el horario de los apoyos, "algunas sesiones son compartidas, de forma que no se pueden hacer dentro del aula, porque el otro alumno igual es de otro curso" (CAS2, GF3, PT2).

La ALE2 y la PT1 también consideran importante garantizar la participación fuera del aula y del centro, por ejemplo a la hora del patio y en las excursiones. La madre añade a esto que "ella todas esas actividades (fuera de la escuela) sí que las hace igualmente de manera inclusiva, dentro, con el resto de los compañeros" (EI3).

El tiempo es una variable relevante para la participación del joven con EPF del CAS3 pues requiere una flexibilización en los horarios, en los exámenes y en objetivos. "Los exámenes si los tiene a principio de semana mucho mejor que al final, por el cansancio de la semana" (EI7, T).

En cuanto al apoyo (C.2.3.), en el CAS1 se intenta que se realice en el aula para que la niña no salga de clase. Dentro del aula, el personal de apoyo y la tutora se coordinan para atender ambas las necesidades del grupo clase. No obstante, se remarca que el papel de la educadora es el de acompañar y no consentir, "ella la acompaña, pero tiene que ser ella (la niña) la que tenga que ir a buscarse el material" (GF1, T). Sin embargo, en momentos concretos el apoyo se realiza fuera del aula, "se prepara a la alumna un poco, sobre todo, en el nivel de la comunicación y lenguaje para que sea capaz, después cuando esté en el aula, de participar activamente y que entienda aquello que se hace" (GF2, PT). Fuera del centro, la niña también recibe el apoyo de logopedia y psicología particular una vez a la semana.

En el CAS2 se valora positivamente que la niña pueda tener momentos de estar en el aula sin personal de apoyo, un progreso respecto a cursos anteriores. Cuando el apoyo se realiza dentro del aula, las educadoras y la logopeda refuerzan las actividades la psicomotricidad y la comunicación de la niña. Fuera del aula, el trabajo es más específico: la fisioterapia, el taller de musicoterapia y el taller sensorial. Este centro potencia el apoyo entre iguales en el aula "los compañeros, por ejemplo, si estamos trabajando la comunicación con PECS (sistema de comunicación de intercambio pictórico), conocen el sistema y también participan en la comunicación" (GF3, ALE1). En el trabajo en equipo también "se encargan sus compañeros de que ella realice su función. Si ella es la repartidora del material, sus compañeros la ayudan" (GF3, ED2). En los desplazamientos por el centro cada vez es un compañero el responsable de acompañarle en las entradas y salidas, y están muy satisfechos de ser los ayudantes. Fuera del centro, la niña asiste a terapia ocupacional, a logopedia y a terapia de caballos y nombran que es fundamental su coordinación con la escuela.

En todos los casos, tal y como afirma la madre del CAS3, el apoyo de la familia es fundamental para trabajar la resiliencia frente a la enfermedad:

"Enseñarles una rutina de autoexigencia, de autocontrol de decir "no puedo estar todo el rato, me duele, me duele, estoy muy cansado"; al revés, distraer la mente en hacer manualidades, pintar, tocar un instrumento...Si la familia no lo tiene muy claro, no refuerza al niño emocionalmente para que se esfuerce" (EI6, M). 


\section{C3. Logro}

La siguiente figura (figura 3) ayuda a ordenar la presentación de los resultados de esta categoría que se articulan en dos subcategorías: evaluación y tránsito, aunque se ha añadido una tercera de carácter emergente sobre expectativas, muy presentes en todas las referencias a los aprendizajes y progresos del alumnado.

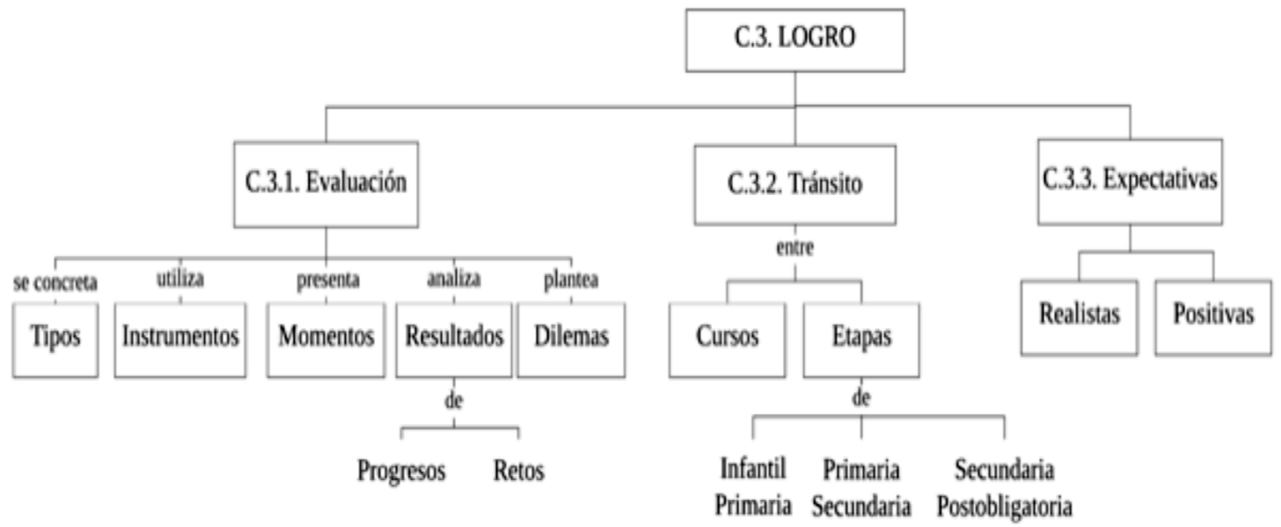

Figura 3. Mapa relacional: logro

La evaluación (C.3.1.) se considera un elemento clave del proceso de inclusión. Es de diferentes tipos y se realizan en diferentes momentos, utilizando variedad de instrumentos. En el CAS1, el personal implicado realiza sesiones de evaluación en las que se comparten percepciones, se revisa la ACIS y se decide qué objetivos se van a priorizar. En cambio, en el CAS2 el aprendizaje se evalúa mediante la escala Denver: "utilizamos la escala Denver para evaluar, de forma que evaluamos a principio de curso, segundo y tercer trimestre, para ver los cambios y añadir ítems. (GF3, PT2). No obstante, la evaluación de los objetivos se hace entre todos en todo momento, mediante la observación.

En cuanto a los resultados evaluados, en el caso 1 y en el 2, se valora el progreso en aspectos más generales como la autonomía, la atención, el esfuerzo, la movilidad y la motivación, y sociales como la comunicación y la mirada. Afirman que "ha avanzado mucho en cuanto a comprensión y a la hora de poder expresarse con sus iguales y con los adultos" (CAS1, GF2, ALE) y también que "estamos notando que hay una progresión, en su autonomía y sobre todo que hay una felicidad enorme en ella cuando viene a la escuela" (CAS2, EI3, M). De igual modo hacen referencia a los retos, expresados más sobre dimensiones generales y sociales que académicas: "Que ella por sí misma pueda tener momentos de trabajo autónomo, de espera, de estar con los compañeros y disfrutando en compañía, y de poder pedir pequeñas cosas que le interesen" (CAS2, GF3, PT1). Las directoras definen el logro como el bienestar social y emocional de las niñas en la escuela: - El grupo clase es muy importante para ella, para su pertenencia y en estos momentos es básico que ella venga contenta a la escuela, esté bien y no tanto lo que aprenda" (CAS1, EI2). 
Sin embargo, en el CAS3 se plantean de nuevo dilemas, en este caso sobre la evaluación. Uno se refiere a la resistencia de algunos profesores a replantear los procesos de evaluación en función de las posibilidades del alumnado y la falta de reflexión crítica de los propios docentes: "la dificultad del alumno en el aprendizaje no solo depende de él, hay que analizar las variables contextuales" (GF5, O). Otro dilema es el dar a conocer o no la enfermedad a los compañeros. En este sentido, la familia y el joven agradecen que desde el centro se haya respetado su petición de no revelar su enfermedad, aunque el propio alumno afirma: "sí, creo que lo entenderían (los compañeros), pero pienso que no es algo a contar a menos que sea necesario" (GF6, A). Para ser el primer curso del joven en este instituto, el tutor menciona como un logro su integración con su grupo clase. El reto que se les presenta es seguir manteniendo una buena comunicación entre el joven, la familia y el profesorado para conocer y atender las ausencias.

En cuanto a la transición (C.3.2.) se refieren al paso de un curso a otro y entre etapas. Para la directora del CAS1 la repetición todavía no es una opción "no consideramos la repetición como una oportunidad aún en estos momentos. Sería posiblemente de cara al último curso considerar... a ver cómo está" (EI2, D). Tienen un plan de transición entre etapas, de la escuela al instituto, y una buena comunicación con el servicio de orientación. Esta es una de las preocupaciones de la madre del CAS2 pues piensa que: "secundaria no funciona como primaria [ ], no está preparada para asumir alguna diversidad funcional, porque no tiene profesionales, recursos ni formación del profesorado" (EI3). Se considera importante en esta transición una buena planificación. En el CAS3, el orientador recomienda a la familia hacer uso de las adaptaciones en las pruebas de acceso a la universidad:“se le puede dar más tiempo en los exámenes y ciertas adaptaciones" (GF5, O).

En cuanto a las expectativas (C.3.3.) sobre el progreso, las madres de los tres casos se muestran precavidas sobre el futuro de sus hijos: "ahora tiene 8 años, acaba aquí y después se va al instituto. Después ya veremos, depende de cómo avance ella, porque yo y mi marido hacemos todo lo que podemos" (CAS1, EI1) y les brindan su apoyo incondicional: "no pretendo que mi hijo sea un genio, pero va a tener una formación" (GF6), añade "nunca le frenaremos" en lo que decida. (CAS3, GF5).

En cambio, las expectativas del personal docente son, en general, más positivas "la niña llegará muy lejos porque lo que pienso es que tiene muchísimas cualidades y es muy feliz y si coge un tema o una profesión que la motive, creo que lo podrá hacer" (CAS1, GF2, ALE).

\section{Discusión y conclusiones}

Los resultados muestran que los procesos que favorecen la presencia del alumnado con EPF se caracterizan por la dedicación de tiempos y espacios a la coordinación del personal implicado: docentes, personal de apoyo, agentes externos si se requiere y las familias. Así, la planificación se considera muy importante para la priorización de los objetivos y la actuación del personal en una misma línea que repercuta en la generalización de los aprendizajes. Este resultado está relacionado con la importancia de planificar sobre un único currículo para todos y todas, como apunta el trabajo de Monzón y Gaintza (2014), ofreciendo las mismas experiencias aunque se deriven aprendizajes diferentes. Se apunta también en los resultados que la variedad metodológica es muy relevante para que todos tengan acceso a este currículo común. Según menciona el pro- 
fesorado, se utilizan múltiples estrategias metodológicas, lo cual va a permitir diferentes formas de acceder, participar y aprender en las aulas. Esto nos lleva a considerar que se están ofreciendo diferentes formas de representar, expresar y comprometerse con el aprendizaje, de acuerdo con la idea de diseño universal de aprendizaje (DUA)(Hitchcock, Meyer, Rose y Jackson, 2002) y de las propuestas metodológicas que permiten la personalización de la enseñanza. Sin embargo, se plantea el dilema de qué hacer cuando los contenidos y estándares de aprendizaje planteados son muy específicos y la alternativa que se ofrece en los casos estudiados es la atención individualizada para desarrollar su autonomía. De acuerdo con Ledesma y Pellejero (2017) una enseñanza flexible, individualizada e integral al alumnado con EPF se considera una buena práctica. De ahí que una primera conclusión del estudio es que la planificación y la coordinación de los agentes implicados es necesaria para orientar las prácticas inclusivas. En función de cómo se planifican y se coordinen los procesos de aprendizaje, el alumnado con EPF podrá acceder, participar y aprender, en mayor o menor medida, en las experiencias de aprendizaje del aula (Gaintza, Ozerinjauregi y Aróstegui, 2018).

En cuanto a la participación, la distribución de los grupos de trabajo busca la heterogeneidad, un factor clave para aprender a vivir con la diversidad (Azorín y Ainscow, 2018). También se prioriza que el alumnado con EPF participe con sus compañeros en diferentes tiempos y espacios (Booth y Ainscow, 2011; Gaintza y Ozerinjauregi, 2017), bien sea dentro del aula, en el patio o las excursiones. Dentro del aula, la posición cercana del alumnado con necesidades, sin estar encima, le permite interactuar en su grupo con normalidad. En ocasiones, las condiciones de la enfermedad exigen una flexibilización en la organización del tiempo adaptada a las ausencias y diferentes ritmos de aprendizaje, en la misma línea que apuntaba el trabajo de Moliner y Sales (2017). En cuanto a los apoyos, la colaboración entre los profesionales se considera fundamental para que tenga lugar dentro del aula siempre que sea posible y esto sería interesante seguir explorándolo con más profundidad desde las modalidades que se establecen en los trabajos de Huguet y Lázaro (2018). Otro elemento clave que se desprende del estudio es el apoyo entre iguales, por ejemplo para acompañar al compañero/a con EPF en los trabajos en equipo y en los trayectos de entradas y salidas y para ello es muy importante que conozcan su sistema de comunicación. No obstante, en algunos casos se requieren apoyos específicos individualizados en el aula, por parte de profesionales no docentes, como educadoras o asistentes, cuyo papel es muy valorado, aunque a veces cuestionado. En este sentido, el trabajo de Verger, Chover y Rosselló (2020) analiza cual es la contribución del/de la auxiliar técnico educativo (ATE) para el desarrollo de una plena inclusión e incide en la necesidad de avanzar hacia la creación de equipos multidisciplinares que trabajen desde la cooperación. Además, las familias recurren en todos los casos a apoyos específicos fuera del centro y del horario escolar. Una segunda conclusión del estudio pone de manifiesto la importancia de la organización de los apoyos en la garantía de la participación plena del alumnado.

Finalmente, en los procesos de aprendizaje, la evaluación es un elemento clave, aunque tanto el progreso como los retos se valoran, no tanto desde la dimensión académica, sino destacando aspectos más generales de socialización, autonomía, bienestar y comunicación. La posibilidad de diversificar la evaluación es, de nuevo, un elemento fundamental, aunque en el trabajo de Orcasitas y Aróstegui (2017) se considera que 
la actitud del tutor va a ser la clave para el avance del alumnado con EPF. En cuanto al tránsito, existe una cierta preocupación por el paso entre etapas. Ello coincide con el resultado del trabajo de Muntaner et al. (2014), al considerar que en secundaria el profesorado suele ser más inseguro en la atención a la diversidad y es el equipo de orientación quien se encarga de la respuesta a este alumnado. Muy interesante resulta la subcategoría emergente de las expectativas de progreso, pues en todos los casos se ha dejado entrever la importancia de creer en la capacidad de aprender del alumnado, tal y como apuntan Booth y Ainscow (2011) aunque los padres se muestran más prudentes que los profesionales en este sentido. Así, la tercera conclusión del estudio pone de manifiesto los dilemas que plantea la evaluación en términos de qué evaluar (aprendizajes académicos-sociales, generales-específicos) y los temores en la transición de la etapa de primaria a la secundaria, lo cual afecta a las expectativas.

A partir de las conclusiones y a pesar de las consabidas limitaciones propias de la metodología de estudio de casos, podríamos establecer como líneas futuras de investigación: analizar qué factores favorecen la inclusión del alumnado con EPF en la etapa de educación secundaria, indagar sobre la coordinación con las figuras de apoyo específico que se contemplan en los recientes decretos de inclusión y profundizar en los procesos de evaluación.

\section{Referencias}

Ainscow, M., Booth, T. y Dyson, A. (2006). Improving Schools, Developing Inclusion. Routledge.

Aróstegui, I. y Monzón, J. (2017). La escolarización de alumnado con enfermedades poco frecuentes en el marco de una escuela inclusiva. En J. Monzón, I. Aróstegui y N. Ozerinjauregi (ed.), Alumnado con enfermedades poco frecuentes y escuela inclusiva (p. 9-18). Octaedro.

Azorín, C. y Ainscow, M. (2018). Guiding schools on their journey towards inclusion. International Journal of Inclusive Education, 1-19. https://doi.org/10.1080/13603116.20 18.1450900

Bartolomé, M. (2017). Diversidad educativa ¿Un potencial desconocido? Revista de Investigación Educativa, 35(1), 15-33. https://doi.org/10.6018/rie.35.1.275031

Bisquerra, R. (2009). Metodología de la investigación educativa. (2ª ed.). La Muralla.

Booth, T. y Ainscow, M. (2011). Index for Inclusion. Developing Learning and Participation in Schools. CSIE.

CEG-RD (Comission Expert Group on Rare Diseases). (2016). Recommendations to support the incorporation of rare diseases into social and policies. https://ec.europa.eu/health//sites/ health/files/rare_diseases/docs/recommendations_socialservices_policies_en.pdf

Echeita, G. y Ainscow, M. (2011). La educación inclusiva como derecho. Marco de referencia y pautas de acción para el desarrollo de una revolución pendiente. Tejuelo, (12), 26-46.

Gaintza, Z. y Ozerinjauregi, N. (2017). Líneas de actuación en la escolarización del alumnado con enfermedades poco frecuentes en la comunidad autónoma del País Vasco. En J. Monzón, I. Aróstegui y N. Ozerinjauregi (ed.), Alumnado con enfermedades poco frecuentes y escuela inclusiva (p. 31-46). Octaedro. 
Gaintza, Z., Ozerinjauregi, N. y Aróstegui, I. (2018). Educational inclusion of students with rare diseases: schooling students with spina bifida. British Journal of Learning Disabilities, 46(4), 250-257. https://doi.org/10.1111/bld.12246

García Parra, M. (2019). Ética y resiliencia familiar para una atención integral en el campo de las enfermedades raras. International Journal of Developmental and Educational Psychology INFAD Revista de Psicología, 2, 271-280,

Generalitat Valenciana. Decret 104/2018, de 27 de juliol, del Consell, pel qual es desenvolupen els principis d'equitat i d'inclusió en el sistema educatiu valencià. Diari Oficial de la Generalitat Valenciana n. ${ }^{-}$8356. https://www.dogv.gva.es/datos/2018/08/07/ pdf/2018_7822.pdf

Gradaille, R. y Caballo, M. B. (2016). Las buenas prácticas como recurso para la acción comunitaria: criterios de identificación y búsqueda. Contextos educativos, (19), 75-88. https://doi.org/10.18172/con.2773

Hitchcock, C., Meyer, A., Rose, D. y Jackson, R. (2002). Providing New Access to the General Curriculum. Universal Design for Learning. TEACHING Exceptional Children, 35(2), 8-17. https://www2.bc.edu/alec-peck/Jackson\%20UDL.pdf

Huguet, T. y Lázaro, L. (2018). Iniciar y mantener prácticas de docencia compartida en las aulas. Aula de innovación educativa, (275), 39-44.

Ledesma, N., y Pellejero, L. (2017). Voces de madres y de menores con enfermedades en la comunidad floral de Navarra. En J. Monzón, I. Aróstegui y N. Ozerinjauregi (ed.), Alumnado con enfermedades poco frecuentes y escuela inclusiva (p. 149-180). Octaedro.

Linertová, R., González-Guardarrama, J., Serrano-Aguilar, P., Posada-De-la-Paz, M., Péntek, M., Iskrov, G. y Ballester, M. (2019). Schooling of Children with Rare Diseases and Disability in Europe. International Journal of Disability, Development and Education, 66(4), 362-373. https://doi.org/10.1080/1034912X.2018.1562159

Ministerio de Sanidad y Política Social. (2009). Estrategia en Enfermedades Raras del Sistema Nacional de Salud. Consejo Interterritorial del Sistema Nacional de Salud. https:// www.mscbs.gob.es/organizacion/sns/planCalidadSNS/docs/enfermedadesRaras.pdf

Moliner, O. y Sales, A. (2017). Informe de situación sobre la escolarización del alumnado con enfermedades poco frecuentes en la Comunidad Valenciana. En J. Monzón, I. Aróstegui y N. Ozerinjauregi (ed.), Alumnado con enfermedades poco frecuentes y escuela inclusiva (p. 181-196). Octaedro.

Monzón, J. y Gaintza, Z. (2014). La inclusión escolar y social de alumnado con efermedades minoritarias. Una oportunidad de innovación y un beneficio para toda la comunidad. Revista de Educación Inclusiva, 7(3), 1-13. https://revistaeducacioninclusiva.es/index.php/REI/article/view/128

Muntaner, J. J., Forteza, D. y Salom, M. (2014). The Inclusion of Students with Chronic Diseases in Regular Schools. Procedia - Social and Behavioral Sciences, 132, 74-79. https://doi.org/10.1016/j.sbspro.2014.04.280

Muntaner, J. J., Rosselló, M. R. y De la Iglesia, B. (2016). Buenas prácticas en educación inclusiva. Educatio Siglo XXI, 34(1), 31-50. http://dx.doi.org/10.6018/j/252521

Orcasitas, J. R. y Aróstegui, I. (2017). Síntesis y concusiones del estudio: la escolarización del alumnado con enfermedades poco frecuentes, avanzando hacia el futuro. En J. Monzón, I. Aróstegui y N. Ozerinjauregi (ed.), Alumnado con enfermedades poco frecuentes y escuela inclusiva (p. 225-236). Octaedro. 
Parrilla, Á. (2010). Ética para una investigación inclusiva. Revista Educación Inclusiva, 3(1), 165-174. https://revistaeducacioninclusiva.es/index.php/REI/article/view/218

Posada, M., Martín-Arribas, C., Ramírez, A., Villaverde, A. y Abaitua, I. (2008). Enfermedades raras. Concepto, epidemiología y situación actual en España. Anales del Sistema Sanitario de Navarra, 31(2), 9-20. doi: 10.4321/s1137-66272008000400002

Solves, J., Arcos, J. M., Páramo, L., Sánchez, S. y Rius, I. (2018). Estudio sobre situación de Necesidades Sociosanitarias de las Personas con Enfermedades Raras en España. (Estudio ENSERio, datos 2016-2017). FEDER y CREER.

Stake, R. E. (2010). Investigación con estudio de casos. (5 $5^{\mathrm{a}}$ ed.). Estudios Morata.

Verger, S. Chover, L., y Rosselló, M.R. (2020) La contribución del/de la auxiliar técnico educativo (ATE) para el desarrollo de una plena inclusión. Aula abierta, 49 (2)171176.https://doi.org/10.17811/rifie.49.2.2020.171-176

Verger, S., De la Iglesia, B., Paz-Lourido, B. y Negre, F. (2017). Informe de situación sobre la escolarización del alumnado con enfermedades poco frecuentes en las Islas Baleares. En J. Monzón, I. Aróstegui y N. Ozerinjauregi (ed.), Alumnado con enfermedades poco frecuentes y escuela inclusiva (p. 119-148). Octaedro.

Fecha de recepción: 6 de octubre de 2020.

Fecha de revisión: 23 de octubre de 2020.

Fecha de aceptación: 7 de febrero de 2021. 